\title{
Instabile Idiome und Turbulenzen des Diskurses in Talkradiogesprächen
}

Das Kapitel meiner Dissertation, das den instabilen Idiomen gewidmet ist, erregte bei Frau Prof. Martine Dalmas viel Interesse und Freude. Ich möchte ihr meine Sympathie und Dankbarkeit für die stets wohlwollende Betreuung zum Ausdruck bringen, indem ich ein paar dieser eigenartigen idiomatischen Formen näher vorstelle.

\section{Einführung}

Gesammelt wurden die in diesem Artikel untersuchten instabilen Idiome in einem Korpus deutscher und französischer Talkradiogespräche. ${ }^{1}$ Ziel der Dissertation war es, idiomatische Einheiten in der mündlichen Interaktion zu beobachten und deren Funktion(en) in der gegenseitigen Verständigung zu identifizieren. Dabei blieb ursprünglich unklar, ob überhaupt und in welchem Umfang instabile idiomatische Einheiten auftreten würden. Die Talkradiogespräche, die untersucht wurden, kennzeichnen sich u.a. dadurch, dass die Sprecher in Echtzeit kommunizieren und demzufolge ihre Beiträge nicht gänzlich vorbereiten, bzw. kontrollieren können. Merkwürdige Formen tauchten also auf. Sie sind insofern von besonderem Interesse, als dass sie es ermöglichen, die Kriterien der Idiomatizität und der Stabilität zu trennen und zu unterscheiden. Wie funktioniert die Idiomatizität, wenn sie nicht anhand stabilen lexikalischen Materials vermittelt wird? Zum ersten stellt sich aber die Frage der Abgrenzung dieser instabilen Idiome: Wie lassen sie sich definieren? In welchem Umfang treten instabile Idiome auf? Im Teil 3 wird ein Überblick über die syntaktischen und semantischen Mechanismen geliefert, die der instabilen Idiome zugrundeliegen. Da diese Trennung der syntaktisch vs. semantisch operierenden Mechanismen nicht völlig befriedigend ist, gerät schließlich das diskursive Umfeld in den Fokus der Auf-

1 Bei der französischen Sendung handelt es sich um die auf Europe 1 ausgestrahlte und von Caroline Dublanche moderierte Libre antenne, bei der deutschen um die auf 1 Live ausgestrahlte, nach dem Moderator benannte Domian-Sendung. Die Aufnahmen gehen auf Dezember 2010 zurück. 
merksamkeit. Es wird im letzten Teil auf die Auswirkungen dieser idiomatischen instabilen Formen geprüft.

\section{2 „Instabile Idiome“: Abgrenzung und Definition}

Im Zentrum meiner Dissertationsarbeit stand die Idiomatizität, sodass Kompromisse mit der traditionellen Definition der Polylexikalität als die Kombination von graphisch getrennten Wörtern, wie etwa bei Burger (2015: 15), eingegangen werden mussten. Idiomatizität geht nämlich über polylexikalische Einheiten hinaus:

\footnotetext{
Das Besondere an unserer Herangehensweise besteht darin, dass wir die Idiome nicht nur im Rahmen der Phraseologie betrachten, d.h. im Rahmen der linguistischen Disziplin, die sich mit den festen Wortkomplexen verschiedener Art befasst, sondern auch als eine besondere Art von bildlichen Lexikoneinheiten. Einerseits gehören die Idiome (aufgrund ihrer Polylexikalität und ihrer Stabilität) zur Phraseologie. Andererseits haben sie (aufgrund ihrer semantischen Besonderheiten) viele Gemeinsamkeiten mit konventionellen Einwortmetaphern und anderen figurativen Einheiten des Lexikons. [...] Idiome sind für uns nicht nur eine Subklasse der Phraseme, sondern auch eine Subklasse der konventionellen bildlichen Lexikoneinheiten. (Dobrovol'skij/Piirainen 2009: 12)
}

Da z.B. Komposita durchaus idiomatisch sein können, wird der Begriff der Polylexematizität bevorzugt, der als die Kombination von Lexemen (auch 'lexikalische Morpheme' genannt) definiert wird. Kombinationen von graphisch getrennten Einheiten, die ein einziges Lexem (oder lexikalisches Morphem) enthalten, wurden im Korpus als monolexematisch eingeordnet. Durch diese neue Unterscheidung tritt die semantische Komplexität der Einheiten in den Vordergrund zuungunsten der Grenze zwischen Morphologie und Syntax. Dem Kriterium der Polylexematizität zufolge tritt das Kompositum in (1) ins Spektrum der Analyse als polylexematische stabile idiomatische Einheit (PSIE), während die Präpositionalphrase in (2) in die Kategorie der monolexematischen idiomatischen Einheiten (MIE) fällt:

$$
\text { ein Stehaufmännchen (sein) }
$$
im Grunde

Was die Idiomatizität angeht, fasst die folgende Definition von Dobrovol'skij und Piirainen (2005: 39f.) meines Erachtens alle Eigenschaften der Idiome zusammen: „Idiomaticity is understood as a semantic reinterpretation and/or opacity and as being closely related to figurativeness.“ 


\subsection{Das Merkmal der Stabilität}

Während es in den ersten Jahrzehnten der Phraseologie-Forschung um die Festigkeit ging, wird heutzutage der Begriff der Stabilität immer häufiger verwendet. Grund dafür ist, dass er mehr zur Relativität einlädt und den unterschiedlichen komplexen Aspekten dieses Phänomens gerechter wird, während der Begriff der Festigkeit und sein französisches Pendant figement ausdrücklich auf einen hohen Grad der Stabilität hinweisen. Hinzu kommt, dass die Stabilität sich nicht auf die lexikalische oder syntaktische Ebene begrenzt, sondern sich u.a. auch in psycholinguistischen und pragmatischen Aspekten widerspiegelt.

In den letzten Jahren hat sich die Einsicht durchgesetzt, dass Phraseme in weit höherem Grade variabel sind, als man das früher annahm. Das gilt zunächst für die alltägliche Sprachverwendung. Wenn man spontane gesprochene Sprache auf Phraseologie hin untersucht, wird man vielfältigste Abweichungen von den in den Wörterbüchern anzutreffenden Nennformen finden. (Burger 2015: 24).

Tatsächlich ist die Konfrontation zur mündlichen Sprachverwendung ein guter Anlass zur Erweiterung der Sichtweite. Da der Begriff der Stabilität der graduellen Natur des Kriteriums besser entspricht, stellt sich viel mehr noch als mit dem der Festigkeit die Frage der Messungs-, bzw. Identifizierungswerkzeuge dieses Merkmals. Wo liegt die Grenze zwischen einer Einheit, die in ihrer stabilen Form (oder einer ihrer stabilen Formen) auftritt, und einer instabilen Einheit? Lexikographische Werkzeuge helfen nicht weiter, da sie meistens nur eine, höchstens ein paar Formen erfassen. Große Korpora haben den Nachteil, sprachliche Daten aus schriftlichen Quellen zu erfassen. Die Nicht-Erscheinung einer der Instabilität verdächtigen Form in einem Korpus heißt daher noch lange nicht, dass diese Form sehr selten ist. Es ist also davon auszugehen, dass Linguisten zurzeit über kein perfektes Werkzeug verfügen, das es ermöglichte, instabile idiomatische Einheiten im Mündlichen zuverlässig zu identifizieren.

Als instabile Idiome gelten also polylexematische Einheiten, die mindestens ein idiomatisches Element enthalten und in einer weder lexikographisch belegten noch gebräuchlichen Form auftauchen. Beispiele dafür wären Einheiten wie „weihnachtsfreie Zone“, „durch tausend Täler von Tränen gehen“, „,jdm. keinen vorheulen wollen“, „,apporter des lumières“, „être écroulé de rire“.2

Die merkwürdigeren idiomatischen Einheiten des Korpus wurden mit unterschiedlichen Wörterbüchern, Korpora- und Internetabfragen abgeglichen, wobei eine gewisse Subjektivität unvermeidlich ist. Ob es sich um Variationen, absichtliche Modifikationen, Ad-hoc-Bildungen, Fehler oder Freudsche Versprecher

2 Diese illustrierenden Beispiele stammen ebenfalls aus dem Korpus. 
handelt, obliegt nicht dem Linguisten, denn entscheidend für diese Unterscheidung ist u.a. die Intentionalität, die wissenschaftlich an einer sprachlichen Erscheinung nicht messbar ist.

\subsection{Instabile Idiome: Umfang}

In einem Korpus, das aus zwölf Stunden Aufnahmen besteht und jeweils ungefähr 5000 types und 65000 tokens enthält, kommt man $\mathrm{zu}$ den folgenden Ergebnissen:

Tab. 1: Umfang der instabilen vs. stabilen idiomatischen Einheiten

\begin{tabular}{lcccccc}
\hline & \multicolumn{3}{c}{ Korpus FR } & \multicolumn{3}{c}{ Korpus DE } \\
\hline $\begin{array}{l}\text { Umfang des Korpus: } \\
\text { jeweils 6 Stunden }\end{array}$ & types & 4930 & \multicolumn{2}{c}{5133} \\
\cline { 2 - 7 } & tokens & 65762 & & 64709 & \\
\hline idiomatische Einheiten insgesamt & 1228 & & 1183 & \\
\hline darunter: & stabil & PSIE & 484 & $39,4 \%$ & 640 & $54,1 \%$ \\
& & MIE & 661 & $53,8 \%$ & 493 & $41,6 \%$ \\
\cline { 2 - 7 } & instabil & PIE & 83 & $6,2 \%$ & 50 & $4,2 \%$ \\
\hline
\end{tabular}

Erwartungsgemäß bilden stabile idiomatische Einheiten die überwältigende Mehrheit der idiomatischen Erscheinungen. Instabile idiomatische Einheiten stellen einen Anteil von ungefähr 5\% der idiomatischen Einheiten insgesamt dar.

Fraglich bleibt aber, ob die Instabilität dieser Einheiten von den Sprechern überhaupt gemerkt wird. Spielt diese idiomatische Instabilität eine Rolle in der konversationellen Interaktion? Gehört sie zu Störungsfaktoren? Wird sie thematisiert? Auf diese Fragestellungen komme ich in einem späteren Teil (4.1.) zurück, denn die der Instabilität zugrundeliegenden Mechanismen sollen in erster Linie charakterisiert werden.

\section{Typen der Turbulenzen}

Zuallererst soll hier die Wahl des Worts Turbulenz erläutert werden. In der Forschung sind andere Termini gängiger, um instabile Einheiten zu bezeichnen. In der deutschen Phraseologie geht es meistens dann um Abweichungen. Die franzö- 
sische Phraseologie benutzt hin und wieder den Terminus défigement, der allerdings die Existenz einer festen Form voraussetzt, von der ausgegangen wird, um auf die neue, „entfestigte“ Form zu kommen. Der Rückgriff auf die Norm scheint unvermeidlich, um die instabilen Einheiten von den stabilen zu trennen. Aber die Norm soll hier als üblicher Gebrauch verstanden werden, und nicht als Regelwerk. Aus der Konfrontation mit einem Korpus konversationeller Interaktionen geht hervor, dass diese instabilen Einheiten eigentlich das Ergebnis der Interaktionskomplexität sind. Bei der mündlichen Interaktion handelt es sich um eine mehrdimensionale und sehr enge Verstrickung von zahlreichen Einflüssen, Parametern und Bedingungen. So soll der Sprachproduzent nicht nur auf die Korrektheit seiner Äußerungen achten, sondern ständig dafür sorgen, dass sein Diskurs verständlich ist und dem entspricht, was er tatsächlich zum Ausdruck bringen will. Erinnerungen werden aufgerufen, Kenntnisse unterschiedlicher Natur werden eingesetzt. Auch muss er darauf achten, dass sein Gesprächspartner aufmerksam ist und seinen Beitrag hören kann. Stets muss er auf bestimmte Signale aufpassen, die darauf hindeuteten, dass sein Gesprächspartner auch gerne antwortete. Und so weiter, und so fort.

Der Diskurs trägt die äußeren Kennzeichen der Turbulenzen, denen er ausgesetzt wird.

Zunächst werden die der Turbulenzen zugrunde liegenden Mechanismen vorgestellt. In unserem Korpus lassen sich die sogenannten Turbulenzen in zwei Kategorien fassen.

\subsection{Syntaktische Turbulenzen}

Die erste Kategorie fasst die Fälle zusammen, welche die syntaktische Ebene betreffen. Verdeutlicht werden die meist festgestellten Turbulenzen hier anhand ausgewählter deutscher und französischer Korpusbelege. ${ }^{3}$

$\mathrm{Zu}$ den syntaktischen Turbulenzen zählt in erster Linie die (syntaktisch) nicht-prototypische Kombination von einem Verb mit einer Ergänzung.

3 Die Belege werden gemäß der Konventionen der Gesprächsanalyse (u.a. ohne Großschreibung und Interpunktion) angegeben. Prosodische Züge werden nur angegeben, wenn sie ausdrücklich kommentiert werden. 


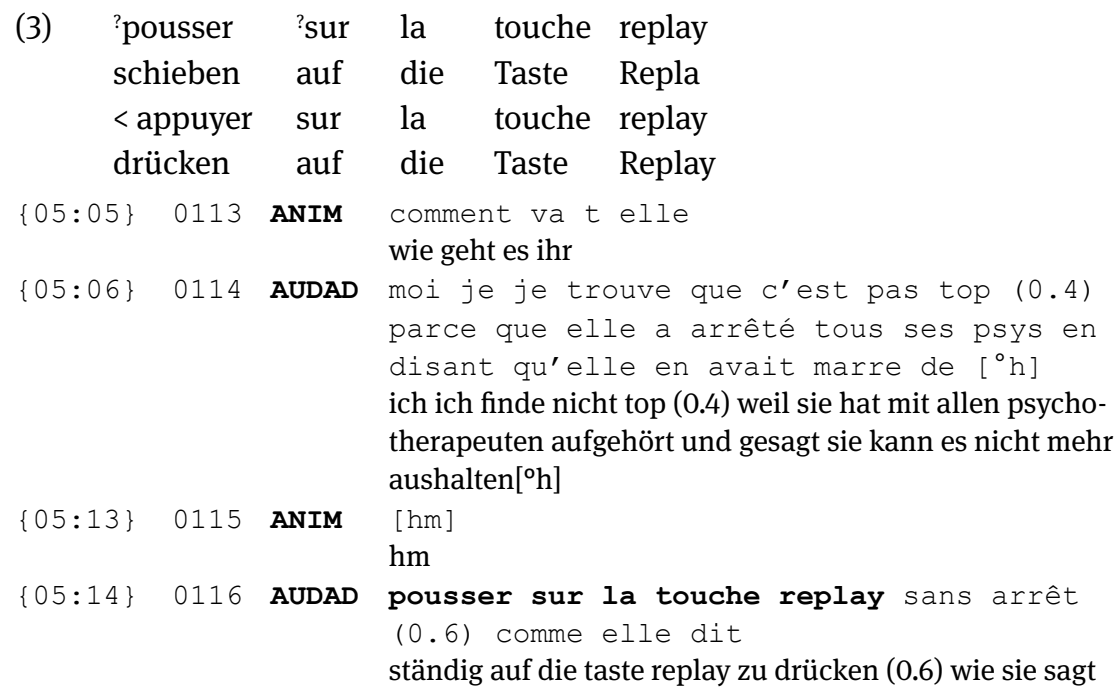

(4) etw. nicht auf den ?Schirm kriegen

$<$ etwas auf dem Schirm haben

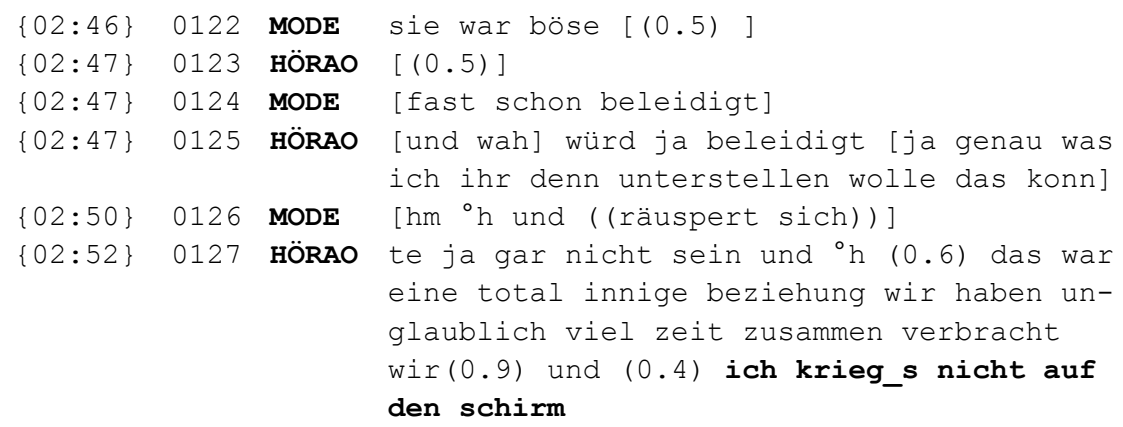

Bemerkenswert bei den Belegen (3) und (4) sowie bei den meisten instabilen Idiomen dieser Kategorie ist die Tatsache, dass der Prozess der Turbulenz völlig nachvollziehbar ist. Bei (4) bildet die Ergänzung des Verbs pousser mit einer Präpositionalphrase mit sur eine Abweichung. Das Verb im Sinne von drücken wird tatsächlich transitiv benutzt. Im Hintergrund dieser syntaktischen Turbulenz steht vermutlich die semantische Nähe des produzierten Verbs pousser mit appuyer. Beide Handlungen erfolgen durch einen von einem animierten Lebewesen ausgeübten Druck, bloß sind die Gegenstände unterschiedlich groß: Wenn es um eine Tür geht, wird man das Verb pousser benutzen, während das Verb appuyer für einen Knopf verwendet wird. Bei (4) lässt sich die Abweichung durch 
einen aspektuellen Unterschied erklären. So wäre etwas (nicht) auf den Schirm kriegen eine imperfektive Variation von einem resultativen Pendant etwas auf dem Schirm haben, ${ }^{4}$ wie etwa bei koexistierenden Redewendungen wie einen dicken Hals kriegen/bekommen vs. einen dicken Hals haben.

Bei einigen Belegen liegt die Vermutung nahe, dass es sich um eine Mischung mehrerer strukturell konkurrierender Redewendungen handelt, wie bei den Belegen (5) und (6).

(5) ?auf etwas nicht klar kommen

$<\underline{\text { mit }}$ etw. klar kommen

? < auf etw. kommen (klar)

$\begin{aligned}\{03: 28\} 0157 \text { HÖRAD } & \text { zum beispiel öh }{ }^{\circ} \mathrm{h} \text { wenn ich jetzt alle zwei } \\ & \text { tage mal zu meinen eltern gehe mal was es- } \\ & \text { sen gehe mit denen ein bisschen mit denen } \\ & \text { zeit verbringe }{ }^{\circ} \mathrm{h} \text { darauf kommt die zum bei- } \\ & \text { spiel absolut nicht klar }\end{aligned}$

(6) jd. setzt jdm. etwas ?auf den Kopf

$<j d$. setzt jdm. etwas in den Kopf

$?<$ jd./etwas stellt etwas auf den Kopf

\begin{tabular}{|c|c|c|c|}
\hline$\{05: 47\}$ & 0250 & MODE & $\begin{array}{l}\text { da ist dein verlobter }{ }^{\circ} \mathrm{h} \text { du pflegst sei- } \\
\text { ne mutter die so schwer krank ist }{ }^{\circ} \mathrm{h} \text { [er }\end{array}$ \\
\hline$\{05: 51\}$ & 0251 & HÖRBI & [ja] \\
\hline$\{05: 52\}$ & 0252 & MODE & $\begin{array}{l}\text { gt dich äh und er ist aggressiv }{ }^{\circ} \mathrm{h} \text { und äh } \\
\text { zwischen euch gibt es keine schönen momen- } \\
\text { te mehr (0.6) äh das sieht nicht nach einer } \\
\text { zukunft aus für euch beide }\end{array}$ \\
\hline $06: 02\}$ & 0253 & HÖRBI & $\begin{array}{l}(0.5) \mathrm{hh}^{\circ} \text { ich ich er ist eigentlich nicht } \\
\text { so er ist eigentlich nicht so ich frag mich } \\
\text { wer ihm so was auf den kopf setzt }\end{array}$ \\
\hline
\end{tabular}

Mit einem Fragezeichen wird in den Belegen (5) und (6) eine zweite Bildung angegeben, die potenziell mit der ersten, intendierten Struktur aufeinandergeprallt

\footnotetext{
4 Semantisch scheint diese Produktion auch etwas merkwürdig, da jdn./etwas auf den Schirm haben im Duden (2013: 649) die folgende Definition zugeteilt wird, die vermutlich nicht die von der Produzentin intendierte ist: ,jmdn., etw. [nicht] wahrnehmen, sich für jmdn., etw. [nicht] interessieren.“
} 
ist. In diesen beiden Beispielen handelt es sich vermutlich um eine rein strukturelle Mischung: Die zweite Redewendung wurde im Gespräch nicht aktiviert und passt von der Bedeutung her nicht in den Kontext.

Andere Abweichungen, die anscheinend aus einer Mischung zweier Redewendungen bestehen, mögen absurd erscheinen, so wie der Beleg (7). In der Interaktion aber ist dieses Aufeinanderprallen nachvollziehbar.

$\begin{array}{cll}\text { (7) tomber } & \text { sur } & \text { un ?mur } \\ \text { fallen } & \text { auf } & \text { einen Maue /eine Wand } \\ \{05: 40\} \quad 0177 & \text { AUDAA } & \text { c'est un peu ausi le con le fin comment } \\ & \text { dire un peu le contraire quoi soit j'y vais } \\ & \text { tête baissée et je vais encore heu pt_être } \\ & \text { tomber heu de sur un mur et puis heu (1.7) } \\ & \text { es ist auch so ein bisschen das ge naja wie soll ich es sagen } \\ & \text { das gegenteil ne entweder ich gehe dahin mit gesenktem } \\ & \text { kopf und ich werde nochmal hm vielleicht fallen hm aus auf } \\ & \text { einen mauer (1.7) }\end{array}$

Der Sprecher AUDAA aktiviert kurz davor die Redewendung tomber de haut, ${ }^{5} \mathrm{um}$ seine Überraschung und Enttäuschung zu bezeichnen. Länger ging es in diesem Gespräch darum, dass AUDAA übereilte Entscheidungen traf. Selbst wenn die produzierte Einheit sehr an die Redewendung tomber sur un os/problème $e^{6}$ erinnert, liegt ihr Zustandekommen wahrscheinlich an einer Kontamination zwischen tomber de haut und foncer dans un/le mur. ${ }^{7}$

Die nächste, öfters auftretende Turbulenz besteht in dem Einfügen einer von der Valenz nicht vorgesehenen Ergänzung. Im Beleg (8) zum Beispiel wird die Redewendung trouver sa voie gegen die vorgesehene Valenz reflexiv verwendet.

$\begin{array}{llll}\text { ?se } & \text { trouver } & \text { sa } & \text { voie } \\ \text { sich } & \text { finden } & \text { seine } & \text { Bahn }\end{array}$

\{01:12\} 0026 AUDAO oui c'est un diplôme j'ai fait pas mal

d'années ça (0.8) actuellement bon je suis

je suis charcutier mais heu

Ja es ist ein diplom ich habe vor ein paar jahren es gemacht

(0.8) heutzutage na ich bin ich bin metzger aber hm

5 Litt: fallen von hoch/oben $\rightarrow$ sehr erstaunt sein; $\approx$ aus allen Wolken fallen.

6 Litt: fallen auf einen Knochen, ein Problem $\rightarrow$ auf ein Problem stoßen.

7 Für eine ausführlichere Erläuterung dieses Belegs siehe Espinat (i.Dr.).

Litt: zurasten auf eine Mauer $\rightarrow$ voreilig und ohne Rücksicht auf Verluste handeln; $\approx$ mit dem

Kopf durch die Wand wollen. 


\begin{tabular}{|c|c|c|c|}
\hline$\{01: 20\}$ & 0027 & ANIM & $\begin{array}{l}\text { ah ça n'a rien à voir } \\
\text { ah es hat damit nichts zu tun }\end{array}$ \\
\hline$\{01: 21\}$ & 0028 & AUDAO & $\begin{array}{l}\text { oui ça a rien à voir et }{ }^{\circ} \mathrm{h} \text { actuellement je } \\
\text { me suis jamais trouvé ma voie réelle } \\
\text { ja es ist völlig anders und hm heutzutage ich hab mir noch } \\
\text { nie meine echte bahn gefunden }\end{array}$ \\
\hline
\end{tabular}

Im folgenden Beleg können verschiedene Turbulenzen aufgedeckt werden:

$\begin{array}{lllll}\text { ?y } & \text { ?mettre } & \text { une } & \text { croix } & \text { dessus } \\ \text { darauf } & \text { stellen } & \text { einen } & \text { Kreuz } & \text { darauf }\end{array}$
\{10:58\} 0289 ANIM il vous dit pas il reste évasif et puis il vous dit ${ }^{\circ} \mathrm{h}$ bon il faut qu'on se voie (0.8) voyez donc même là il est fuyant [(0.6)] er sagt es ihnen nicht er bleibt in der schwebe er sagt ihnen ${ }^{\circ} \mathrm{h}$ na wir müssen uns treffen (0.8) sehen sie dann sogar da ist er ausweichend (0.6)

$\{11: 04\} 0290$ AUDAJ [(0.6)] oui puis alors il me dit heu bon je vais vendre ma mai ma maison ${ }^{\circ} \mathrm{h}$ heu je vais heu puis alors je lui dis mais enfin tu bon réfléchis y a ton fils ${ }^{\circ} h$ oh mais mon fils $j^{\prime} y$ mets une croix dessus

(0.6) ja und dann er sagt zu mir hm ich werde mein $\mathrm{h} h$ haus ${ }^{\circ} \mathrm{h} h \mathrm{~h}$ je werde hö und dann sage ich ihm aber du überleg es dir es gibt ja deinen sohn ${ }^{\circ} \mathrm{h}$ ach aber mein sohn ich stelle drauf einen kreuz drauf

Zum ersten wird die Valenz durch das Pronomen $y$ ergänzt, das hier überflüssig ist, zumal dessus schon als Wiederaufnahme der präpositionalen Ergänzung sur qqc. gilt. Außerdem sind zwei andere Turbulenzen identifizierbar. Das Verb mettre anstatt von faire rechtfertigte die Einordnung in die Kategorie der nichtprototypischen Kombination Verb-Ergänzung. Hinzu kommt, dass die Konstruktion es theoretisch nicht zulässt, diese Redewendung mit animierten Lebewesen als Ergänzung der Präpositionalgruppe zu verwenden.

Der letzte, im Korpus relativ produktive Turbulenztyp syntaktischer Natur operiert eigentlich an der Schnittstelle von Syntax und Semantik. Dabei wird eine Ergänzung durch eine andere unerwartete Ergänzung ersetzt. Eine der Turbulenzen, die im Beleg (9) identifiziert wurde, illustriert schon diesen Mechanismus. Er sei hier mit einem weiteren Beleg geschildert. Im folgenden Korpusauszug wird die idiomatische Lesart eines Verbs aktiviert, das mehrere Konstruktionen akzeptiert. In einem ersten Zug wird die idiomatische Struktur in ihrer stabilen Form verwendet (siehe unterstrichene Stelle). 
?ein Haus verlieren

< einen Freund, einen Angehörigen verlieren

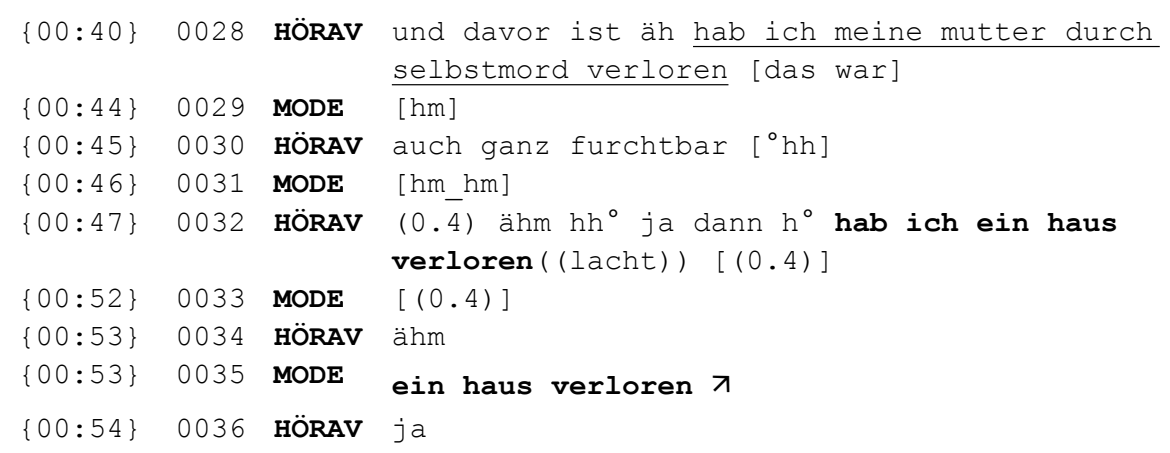

Wenn das Verb verlieren kurz danach vom Sprecher HÖRAV wieder verwendet wird, stößt es auf Erstaunen beim Moderator (siehe steigende Intonation, Segment $\mathrm{n}^{\circ} 35$ ). In welcher Lesart soll hier das Verb verstanden werden? Nicht-idiomatisch kann man tatsächlich einen Gegenstand verlieren, wie zum Beispiel seine Schlüssel oder sein Handy. Lässt es die wörtliche Lesart zu, dieses Verb mit einem Gegenstand wie ein Haus zu kombinieren? Ist die idiomatische Lesart die vom Produzenten intendierte, dann bleibt die Ergänzung durch Haus genauso merkwürdig. Das Verb verfügt über zwei idiomatische Lesarten: Es kann als Euphemismus für sterben gelten, oder die Bedeutung von etwas nicht mehr genießen haben, wie etwa bei das Vertrauen von jdm. oder den Verstand verlieren. Im Fall der ersten idiomatischen Bedeutung fehlt es Haus an den notwendigen semantischen Zügen (+ animiert, + sterblich). Für die zweite Interpretation stört Haus insofern, als dass es einen konkreten Gegenstand bezeichnet. In beiden Fällen ist eine Ergänzung mit einem Substantiv wie Haus in der Valenz der idiomatischen Lesart des Verbs nicht vorgesehen. Obwohl der Moderator durch die Wiederholung der Einheit signalisiert, dass sie einem auffällt und potenziell korrigiert werden sollte, bleibt HÖRAV bei seiner ursprünglichen Formulierung.

\subsection{Semantische Turbulenzen}

Wie wir gerade feststellen konnten, ist die Grenze zwischen Syntax und Semantik teilweise durchlässig, sodass einige instabile Einheiten in beide Kategorien der Turbulenzen fallen mögen. Im Folgenden werden die Typen vorgestellt, die relativ eindeutig semantischer Natur sind. Zwei Subklassen der semantischen Turbulenzen sind besonders produktiv im Korpus. Die erste Subklasse bezeugt eine 
enge Verwandtschaft mit dem syntaktischen Turbulenztyp, der gerade erläutert wurde. Die Gemeinsamkeit liegt darin, dass die erwartete Ergänzung durch eine merkwürdige ersetzt wird. Im Unterschied zum verwandten, syntaktischen Typ unterhalten die hier unerwarteten Ergänzungen ein engeres semantisches Verhältnis zur erwarteten Variante. Zwischen beiden besteht eine hypero-/hyponymische, holo-/meronymische, synonymische oder metonymische Beziehung.

(11) kein Bein auf ?den Boden kriegen

$<\equiv$ kein Bein auf die Erde kriegen

\begin{tabular}{|c|c|c|c|}
\hline $6: 17\}$ & 0279 & MODE & $\begin{array}{l}\text { glaub ich auch wenn die dir jetzt gesagt } \\
\text { hätte pass mal auf damals äh du bist zu } \\
\text { zickig oder zu nachtragend oder du bist zu } \\
\text { lahm im bett oder so }{ }^{\circ} \mathrm{h} \text { da kann man ja was } \\
\text { mit anfangen (0.6) wenn es einem auch weh } \\
\text { tut aber kann man da kann man was mit an- } \\
\text { fangen aber so wenn sie sagt war super und } \\
\text { ganz toll und }{ }^{\circ} \mathrm{h}[(0.9) \text { ] }\end{array}$ \\
\hline$\{06: 31\}$ & 0280 & HÖRAO & $\begin{array}{l}\text { [(0.9)] sie hätte das halt mitgenommen weil } \\
\text { es ein schönes gefühl war und hätte gedacht } \\
\text { das hat mit mir überhaupt nichts zu tun } \\
\text { egotusse }\end{array}$ \\
\hline $06: 38\}$ & 0282 & HÖRAO & $\begin{array}{l}\text { ja (0.7) ja aber das kriege ich nicht über- } \\
\text { ein mit der person [die ich ge] }\end{array}$ \\
\hline $6: 42\}$ & 0283 & MODE & {$[\mathrm{hm} \quad]$} \\
\hline $6: 42\}$ & 0284 & HÖRAO & heiratet hab [die ich ] \\
\hline $06: 43\}$ & 0285 & MODE & {$[\mathrm{hm}]$} \\
\hline$\{06: 43\}$ & 0286 & HÖRAO & geglaubt hab zu kennen \\
\hline $06: 44\}$ & 0287 & MODE & \\
\hline $06: 45\}$ & 0288 & HÖRAO & $\begin{array}{l}\text { ich kriege natürlich kein bein auf den } \\
\text { boden }\end{array}$ \\
\hline
\end{tabular}

In diesem Beleg liegt eine metonymische Beziehung der Turbulenz zugrunde, da der Boden tatsächlich eine Kontiguität mit der Erde aufweist. Bei manchen Idiomen sind übrigens beide Substantive zulässig (z.B. mit beiden Beinen/Füßen (fest) auf der Erde/auf dem Boden/im Leben stehen), während andere idiomatische Bildungen diese Konkurrenz nicht ermöglichen (z.B. bodenständig/*erdeständig sein, am Boden/ ${ }^{*}$ auf der Erde liegen).

Die zweite Subklasse der semantischen Turbulenzen besteht aus Redewendungen, die außerhalb des Kontexts schwer verständlich wären und/oder mit einer anderen förmlich verwandten aber semantisch nicht passenden Redewendung verwechselt würden. 
?mit einem Ohr hängen

₹ die Ohren hängen lassen

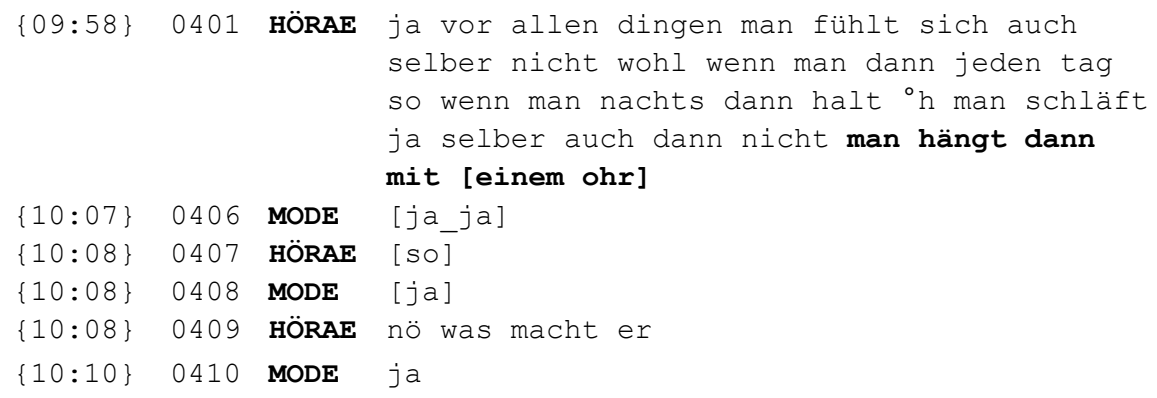

Im Gespräch, aus welchem der Beleg (12) entnommen wurde, erzählt HÖRAE, dass ihr Lebensgefährte psychisch krank ist. Sie erzählt ihr erschöpfendes Leben und ihre Angst um ihren Mann. Das produzierte Idiom mag an der Redewendung die Ohren hängen lassen erinnern, hat aber mit deren Bedeutung wenig zu tun. Im Kontext versteht man nämlich, dass mit einem Ohr hängen eher mit die Ohren spitzen/aufmachen verwandt ist: Es geht um die ständige notwendige Wachsamkeit der Sprecherin, die auf jedes Geräusch horchen muss. Das Idiom die Ohren spitzen/aufmachen impliziert eine intendierte Handlung, wie die Definition des Duden (2013: 546) „aufmerksam lauschen“ bestätigt. Dagegen erhalten viele Ausdrücke mit dem Verb hängen den semantischen Zug der Passivität, ja sogar einer durch die Erschöpfung entstandenen Passivität. Selbst wenn diese Kombination nicht attestiert ist und sicherlich selten ist, entspricht sie vielleicht am nächsten dem, was die Sprecherin ausdrücken möchte.

\subsection{Fazit}

Aus diesem Überblick geht hervor, dass syntaktische und semantische Mechanismen bei der Entstehung instabiler Idiome meistens schwer zu trennen sind. Nicht alle idiomatischen instabilen Einheiten können eindeutig der einen oder anderen Kategorie zugeordnet werden. Es liegt hauptsächlich an ihrer lexikalischen Komplexität. Von welcher Struktur wird denn ausgegangen? Intendierte die Sprecherin im Beleg (12) eine Konstruktion mit hängen? Oder ist sie von einer Redewendung, die das Bild des Ohrs enthielt, ausgegangen? Gab es überhaupt eine klare Ausgangsstruktur? Die Analyse der instabilen Idiome ist deswegen äußerst schwierig, weil man entweder auf das Kriterium der Intentionalität zurückgreift, das ja weder messbar noch zuverlässig ist, oder man muss die Reihenfolge der Elemente als Maßstab nehmen. Tut man dies, geht man implizit davon aus, dass 
die Äußerungen Wort für Wort aufgebaut werden, was eine genauso verwirrende Herangehensweise bildet.

Der Versuch, die syntaktische und semantische Ebenen $\mathrm{zu}$ trennen, lässt aber eine wichtige Eigenschaft der mündlichen Interaktion völlig außer Acht, und zwar die Tatsache, dass der Diskurs selber sehr instabil ist und ständig von dem einen oder anderen Parameter beeinflusst wird. Aus diesem Grund wurde die Metapher der Turbulenz verwendet. Genauso wie ein Flug meistens ohne Turbulenzen verläuft, ist das idiomatische Material relativ stabil, sei es auf lexikalischer, pragmatischer oder psycholinguistischer Ebene. Der Beweis dafür ist die geringe Zahl der instabilen Idiome insgesamt im Korpus. Nun mag es passieren, dass die relative Stabilität des Diskursstroms an bestimmten Stellen gefährdet wird, sodass auch dieses Material dann instabil wird. Wie der kurze Überblick über das diskursive Umfeld in 2.2 es vermuten lässt, fällt diese Instabilität auf.

\section{Auswirkungen auf die Interaktion}

Nun soll das diskursive Umfeld eingehender untersucht werden, da es Schlüssel zur Auffälligkeit und zur Art und Weise liefert, wie diese instabilen Formen in der Interaktion gehandhabt werden.

\subsection{Phänomene des diskursiven Umfelds: Überblick}

Das Korpus wurde auf Phänomene des diskursiven Umfelds hin untersucht. Paraphrasen, metadiskursive Kommentare, Wiederholungen, Intensitätsmarker, korrigierende und zustimmende Äußerungen im Umfeld der idiomatischen Einheiten wurden annotiert. ${ }^{8}$ Folgende Ergebnisse wurden erzielt:

8 Dabei soll auf die Verwandtschaft der einen Kategorie mit anderen hingewiesen werden. Einige Paraphrasen zum Beispiel grenzen an der korrigierenden Äußerung, während metadiskursive Kommentare als Zustimmung gelten mögen. Einer Äußerung konnten daher mehrere Annotationen zukommen. 
Tab. 2: Anzahl und Ausmaß der Phänomene des diskursiven Umfelds bei instabilen vs. stabilen Idiomen

\begin{tabular}{|c|c|c|c|c|c|c|}
\hline & & & \multicolumn{2}{|l|}{ Korpus FR } & \multicolumn{2}{|c|}{ Korpus DE } \\
\hline \multicolumn{3}{|c|}{ idiomatische Einheiten insgesamt } & 1228 & & \multicolumn{2}{|c|}{1183} \\
\hline \multicolumn{3}{|c|}{$\begin{array}{l}\text { Idiomatische Einheiten in Verbindung mit } \\
\text { einem Phänomen des diskursiven Umfelds }\end{array}$} & 698 & & \multicolumn{2}{|c|}{518} \\
\hline \multirow[t]{3}{*}{ darunter: } & stabil & PSIE & 307 & $63,4 \%$ & 315 & $49,2 \%$ \\
\hline & & MIE & 316 & $47,8 \%$ & 176 & $35,7 \%$ \\
\hline & instabil & PIE & 75 & $90,3 \%$ & 27 & $54 \%$ \\
\hline
\end{tabular}

Über die Hälfte (im deutschen Korpus) und bis zu 90\% (im französischen) der instabilen Idiome sind mit einem der oben aufgelisteten Phänomene verbunden. Die Sensitivität des diskursiven Umfelds geht aus den Daten hervor und weist auf eine Auffälligkeit der instabilen Idiome in der Interaktion. Wie und anhand welcher Phänomene sich diese Auffälligkeit widerspiegelt, verdeutlicht die folgende Grafik:

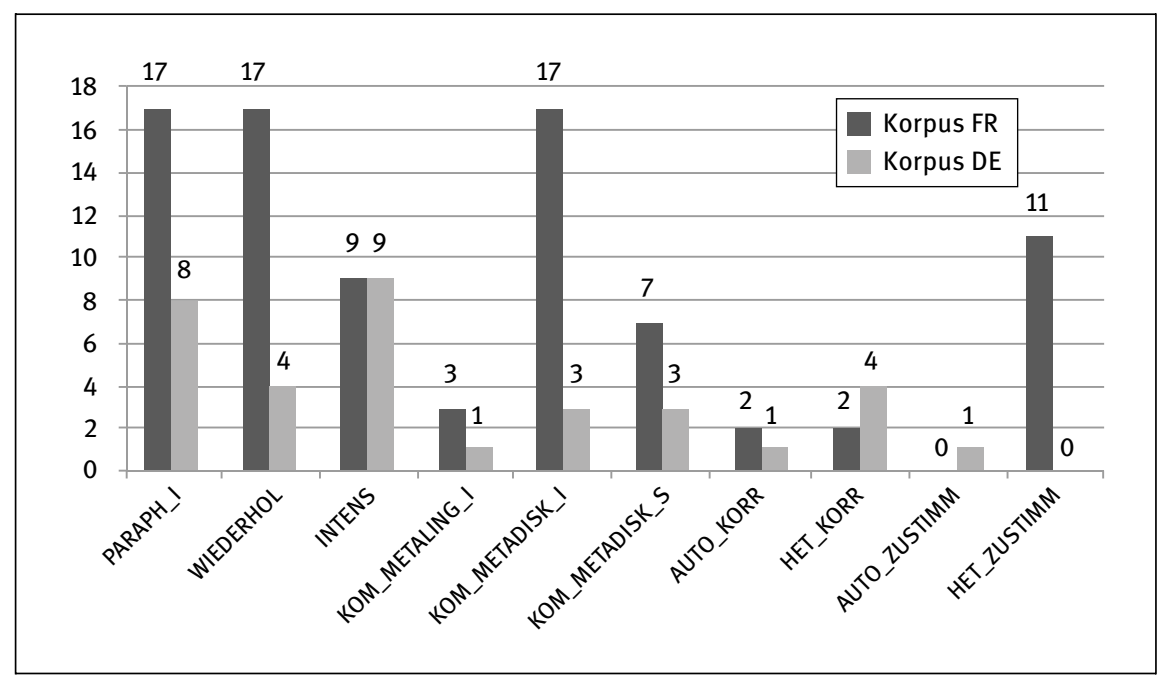

Abb. 1: Verteilung der Phänomene des diskursiven Umfelds instabiler Idiome nach Typen

Die Diskrepanz bestimmter Phänomene zwischen französischem und deutschem Korpus lässt sich durch die Struktur und die Tonalität der jeweiligen Sendung 
erklären. Die Moderatorin der französischen Sendung ist diplomierte Psychologin und gewährt der Entfaltung der Erzählung und des Gefühlsausdrucks mehr Freiraum als der Moderator der deutschen Sendung. ${ }^{9}$ Aus diesem Grund ist es nicht verwunderlich, dass Phänomene des diskursiven Umfelds, die auf eine gewisse diskursive Bemühung deuten, wie etwa metadiskursive Kommentare und Paraphrasen, bei der französischen Sendung viel öfter vorkommen. Auch die fremdinitiierten ${ }^{10}$ zustimmenden Äußerungen sind fast exklusiv der französischen Moderatorin zuzuschreiben.

Um diese relativ verschiedenen Ergebnisse $\mathrm{zu}$ verfeinern, erweist sich der Vergleich mit der Verteilung der Phänomene bei den stabilen idiomatischen Einheiten als notwendig. Somit gewinnt man einen Einblick in die Spezifizität der instabilen Idiome.

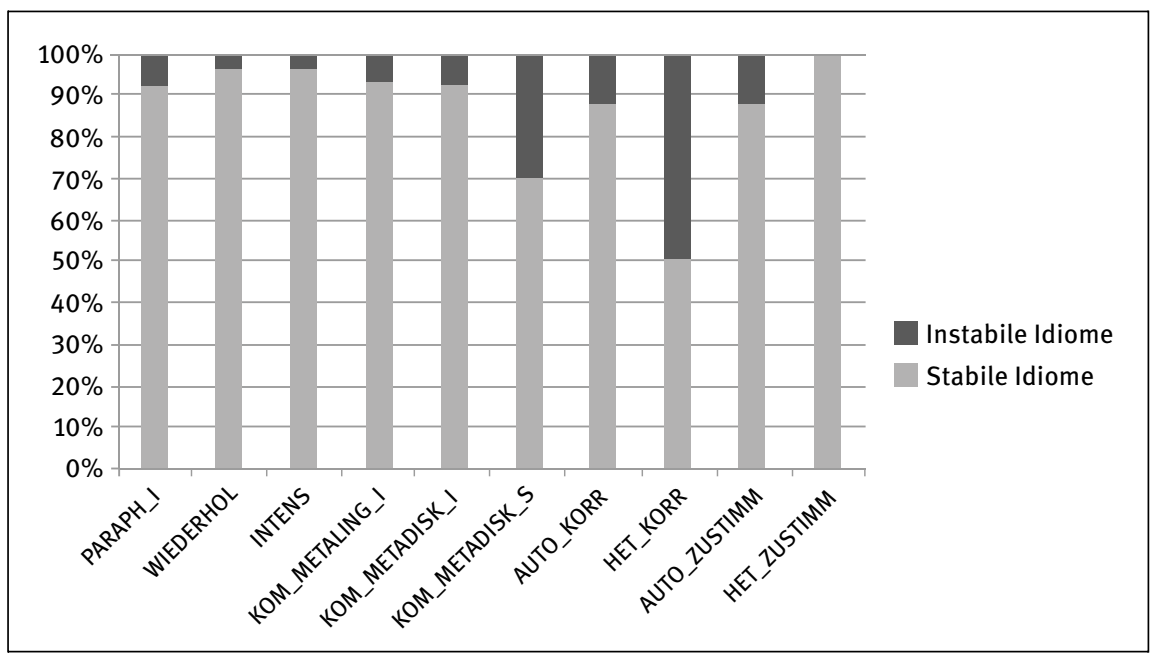

Abb. 2: Diskursives Umfeld der instabilen Idiome: Proportionaler Vergleich mit den stabilen Idiomen - Korpus DE

9 Repräsentativ dafür ist die durchschnittliche Länge der Austausche, die in der französischen Sendung 18 Minuten und in der deutschen 9 Minuten beträgt.

10 „Selbstinitiiert“ heißt, dass das Phänomen vom selben Sprecher produziert wird, wie die idiomatische Einheit. Mit „fremdinitiiert“ wird gemeint, dass der Gesprächspartner/Rezipient des Idioms die Äußerung geäußert hat, in der ein Phänomen des diskursiven Umfelds beobachtet wird. 


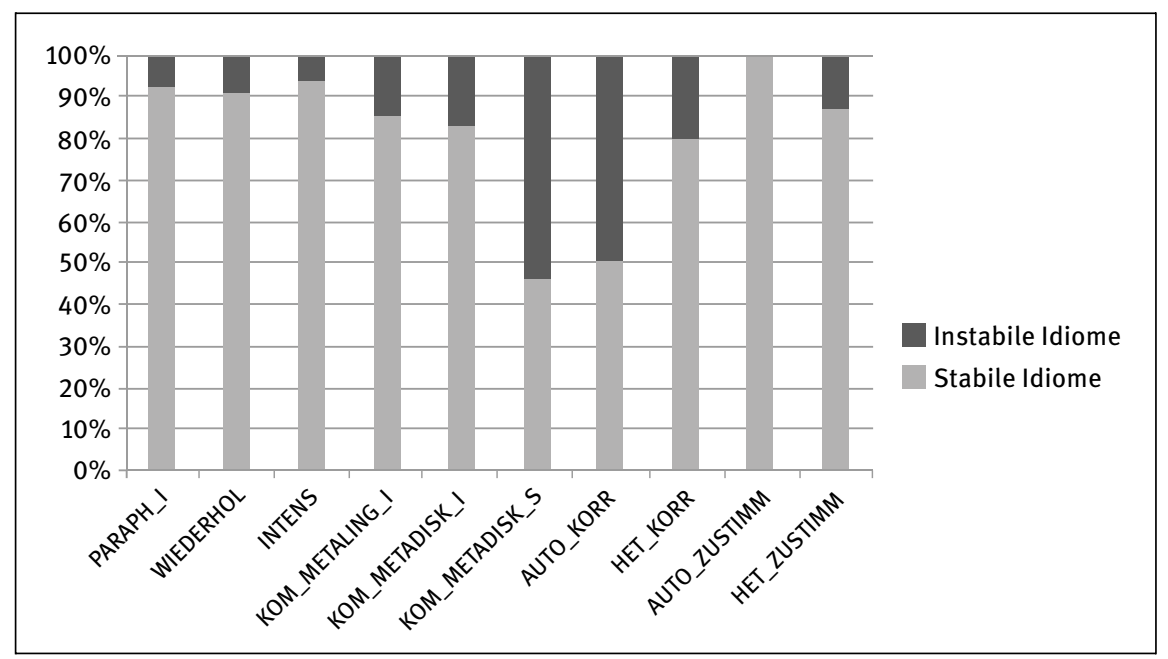

Abb. 3: Diskursives Umfeld der instabilen Idiome: Proportionaler Vergleich mit den stabilen Idiomen - Korpus FR

Aus diesen beiden Grafiken geht hervor, dass Korrekturphänomene, die im Korpus selten sind, proportional öfter im Umfeld eines instabilen Idioms auftreten. Diese Erkenntnis scheint nicht erwartungswidrig. Nichtsdestotrotz lassen sich interessante Schlüsse ziehen. Es zeigt unter anderem, dass diese instabilen Idiome bemerkt werden und als nicht-prototypisch von den Sprechern selbst eingestuft werden.

Auch die metadiskursiven Kommentare zeigen proportional eine Affinität zu den instabilen Idiomen. Unterschieden wurde zwischen:

- metadiskursiven Kommentaren, die die Idiomatizität thematisieren (KOM_METADISK_I), ${ }^{11}$

- metadiskursiven Kommentaren, die die Stabilität thematisieren (KOM_METADISK_S), ${ }^{12}$

11 Repräsentativ für metadiskursive Kommentare, die die Idiomatizität thematisieren, sind Äußerungen, die zu einer näheren Betrachtung oder Erklärung eines idiomatischen Elements veranlassen. Demzufolge gehören fast alle metadiskursiven Kommentare im Umfeld eines Idioms zu dieser Kategorie. Prototypisch wären aber kommentative Äußerungen, wie „dieses Bild, das Sie verwenden“, „,im übertragenen Sinne“ oder „im wahrsten Sinne des Wortes“.

$12 \mathrm{Zu}$ den metadiskursiven Kommentaren, die die Stabilität thematisieren, zählen kommentative Äußerungen, bei denen die (Un-)Gebräuchlichkeit der Formel durchscheint. Als Beispiele können folgende Kommentare genannt werden: ,auch wenn man so sagt“, „kann man sagen“. 
- metalinguistischen Kommentaren, sofern ein metalexikalisches Element vorhanden war (KOM_METALING_I). ${ }^{13}$

Die überproportionale Vertretung der Korrekturen einerseits und der metadiskursiven Kommentare über die Stabilität andererseits ist nicht weiter erstaunlich. Sie bedarf jedoch weiterer Erläuterungen.

\subsection{Spezifizitäten des diskursiven Umfelds: Metadiskursive Kommentare und korrekturähnliche Phänomene}

Was die metadiskursiven Kommentare angeht, übernehme ich die Klassifikation von Techtmeier (2001: 1454), die über die Thematisierungsfunktion dieser Kommentare hinaus, eine weitere, sogenannte Stützungsfunktion und deren Subklassen auftauchen lässt:

Ihre Stützungsfunktion bezieht sich auf

- das Verstehen der jeweiligen Bezugsäußerung bzw. eines globaleren interaktiven Geschehens,

- die Akzeptanz der jeweiligen Bezugsäußerung bzw. eines globaleren interaktiven Geschehens,

- die Ausführbarkeit der vom Sender erwünschten Handlung.

Selbst wenn die Anzahl der metadiskursiven Kommentare im Umfeld der instabilen Idiome sehr gering ist und diese Daten darum relativiert werden müssen, betreffen fast sämtliche Kommentare ${ }^{14}$ die Akzeptanz der Äußerungen, nicht aber

13 Metadiskursiv wird hier hyperonymisch verwendet, da bei allen Kommentaren ein Metadiskurs produziert wird. Was in dieser Arbeit als „metalinguistisch“ gilt, ist also de facto auch metadiskursiv. Dieser zusätzliche Stempel dient dazu, die metadiskursiven Kommentare, in denen (wenn auch laienhafte) Metalexik benutzt wird, zu differenzieren. Beispielhaft für metalinguistische Kommentare in dem hier intendierten Sinne sind Äußerungen, bei denen eine Benennung wie „die(se) Redensart“, „die(se) Redewendung“, usw. erfolgt.

14 Techtmeier (2001: 1457) betont die sehr wahrscheinliche Polyfunktionalität der Kommentare: „[...] man [kann] im Falle einer polyfunktionalen metakommunikativen Äußerung von einer unterschiedlichen Gewichtung einzelner Funktionen ausgehen: eine Unterscheidung in Hauptund Nebenfunktionen scheint daher gerechtfertigt“. Um die Ergebnisse nicht zu verfälschen, wurde jeder Kommentar mit einer einzigen Annotation versehen, welche auf eine sehr deutlich erkennbare Hauptfunktion hinweist. Im Falle einer unklaren Diskriminierung wurde der Kommentar „?“ annotiert.

Im deutschen Korpus sind nur drei metadiskursive Kommentare im Umfeld der instabilen Idiome zu finden, die alle die Thematisierung der Akzeptanz betreffen. Im französischen Korpus 
ihr Verstehen. Auffällig sind sie also nicht, weil sie schwer verständlich sind. Nur die merkwürdige Formulierung erweckt die Aufmerksamkeit der Sprecher.

Die überwältigende Mehrheit der metadiskursiven Kommentare im Umfeld der instabilen Idiome ist selbstinitiiert. Dennoch ist dieser Anteil dem der Kommentare im Umfeld stabiler Idiome sehr ähnlich. Dieses Phänomen zeugt von einer starken Tendenz zur Kooperation in der Nähe der Idiome im Allgemeinen und ist als eine Konsequenz der Idiomatizität zu deuten.

Korrekturen kommen an sich mit jeweils 4 und 5 Fällen für 83 und 50 instabile Idiome selten vor. Es liegt in erster Linie an der negativen Wirkung auf die Interaktion und die faces $^{15}$ der Sprecher. Außerdem soll auf weitere Schwierigkeiten der Diskriminierung verwiesen werden. Die unterschiedlichen Kategorien überschneiden sich nämlich oft, sodass eine Wiederholung der Korrektur der Äußerung dienen mag. Gegebenenfalls wurden die Äußerungen mit mehreren Annotationen versehen. Zweitens ist nicht die tatsächliche Korrektur der Einheit für die Einstufung als Korrektur entscheidend, sondern das Signal, dass das kommunikative Ziel nicht erreicht wurde und daher eine tatsächliche Korrektur oder eine nähere Erklärung wünschenswert sind. Ein solcher Fall lag schon im Beleg (10) vor, als der Moderator das instabile Idiom mit steigender Intonation wiederholte.

Im französischen Korpus wurden zwei fremdinitiierte und zwei selbstinitiierte Korrekturen vorgefunden. Jeweils eine Korrektur bestand in der tatsächlichen Berichtigung des instabilen Idioms, während die andere durch ein Signal der wünschenswerten Erläuterung erfolgte. Im deutschen Korpus wurden vier fremdinitiierte und eine selbstinitiierte Korrektur aufgelistet. Auch hier erfolgte die Hälfte der fremdinitiierten Korrekturen durch eine tatsächliche Berichtigung des Idioms und die andere Hälfte durch ein Signal des verfehlten kommunikativen Ziels. Aufgrund der äußerst geringen Menge der Okkurrenzen werden Schlüsse anderen, umfangreicheren Untersuchungen diesbezüglich überlassen.

\subsection{Fallstudie: „der große Parkplatz von Köln“}

Anhand des folgenden Belegs soll die Verstrickung der Phänomene verdeutlicht werden. Ausnahmsweise handelt es sich um eine Bildung, von der man mit hun-

verfügen wir über 19 Okkurrenzen. Darunter betreffen 14 eindeutig die Akzeptanz, 1 das Verstehen und 4 wurden mit ? annotiert.

15 Siehe face threatening acts in Brown/Levinson (1987: 65) in Anlehnung an Goffman (1967). 
dertprozentiger Sicherheit behaupten kann, dass sie intendiert und als Scherz konzipiert wurde. ${ }^{16}$

(13) der große Parkplatz von Köln

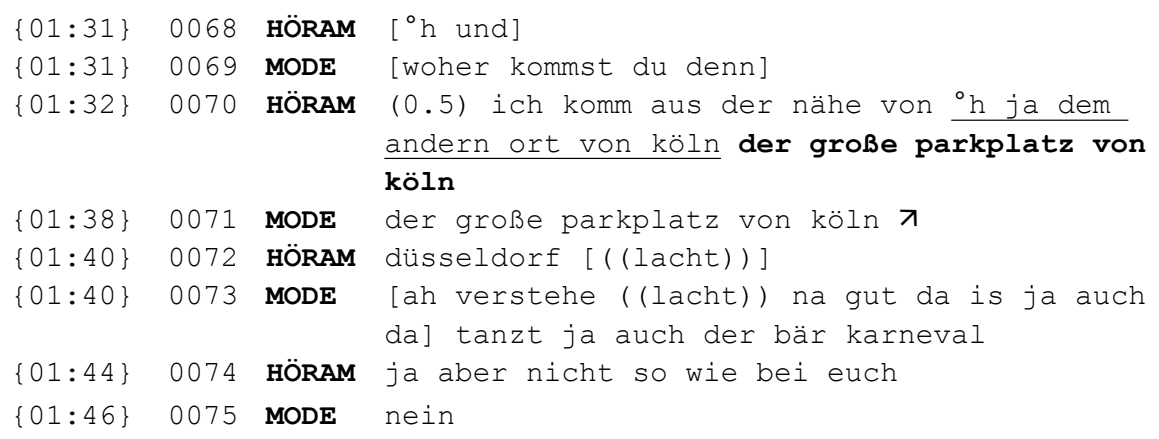

Dieser Auszug entstammt den ersten Minuten des Austauschs. HÖRAM unterbricht den ersten Zug seiner Antwort mitten in einer Präpositionalphrase (siehe die punktiert unterstrichene Stelle des Segments 70) und bereitet seine scherzhafte idiomatische Bildung vor, indem er sie sozusagen vorparaphrasiert ${ }^{17}$ (siehe unterstrichene Stelle). Damit versucht er auf die Nähe und die Rivalität zwischen beiden Städten, Köln und Düsseldorf, anzuspielen. Die Ad-hoc-Bildung und Höhepunkt des Scherzes der große Parkplatz von Köln folgt unmittelbar. An sich zeugt diese Bildung von einer unbestreitbaren Kreativität. Impliziert werden:

- die geografische Nähe, denn ein Parkplatz säumt im Prinzip das Gebäude, zu dem er gehört,

- die mangelnde Attraktivität der Stadt, die als Parkplatz gilt. Sie wird in einer rein zweckhaften Funktion wahrgenommen. Damit wird die Idee hervorgerufen, dass Düsseldorf eine Stadt ist, in der alle, die nach Köln wollen, nur parken. Die erwähnte Größe des Parkplatzes verstärkt die unpersönliche Wirkung.

16 Durch seine Tendenz zur scherzhaften Selbstinszenierung erhielt dieser Sprecher in Espinat (2015) den Titel des „Scherzkeks“, da es sich um den einzigen Anrufer handelte, der sich (mehr oder weniger gute) Wortspiele und (mehr oder weniger gelungene) Scherze zutraute.

17 Theoretisch gilt die nachstehende Äußerung als Paraphrase der ersten. Hier ist aber die Wahrscheinlichkeit groß, dass dem anderen Ort von Köln schon auf der große parkplatz von köln abzielt. 
Obwohl der Moderator Jürgen Domian ein Kölner ist, versteht er die Anspielung auf Düsseldorf nicht. Er wiederholt also diesen Teil der Äußerung mit steigender Intonation als Signal des Unverständnisses, was mit einer Korrektur gleichzusetzen ist. Es zwingt HÖRAM zur Korrektur seiner Äußerung und zur Aufklärung seines Scherzes. Das Lachen von HÖRAM ist übrigens Teil dieser Aufklärung, denn es fungiert als Signal, dass es sich um eine intentionelle Bildung handelte und dass es lustig war, bzw. sein sollte.

Dieser Beleg illustriert mit einem Hauch von Heiterkeit ein allen Erscheinungen instabiler sowie stabiler Idiome zugrundeliegendes Prinzip, und zwar das der Kooperation (Grice 1975). Ob stabil oder nicht, geht es den Sprechern darum, dass die Interaktion möglichst erfolgreich verläuft und ihre faces geschützt werden. Der weitere Verlauf des Austausches zeigt, dass eine Versöhnung dieser unangenehmeren Klärung unmittelbar folgt: Domian spielt auf die gemeinsame Tradition des Karnevals an und erkennt sogar, dass es in Düsseldorf auch eine gute Karnevalstimmung gibt (da tanzt auch der Bär Karneval). Die Klärung des instabilen Idioms der große Parkplatz von Köln hätte den weiteren Verlauf der Interaktion beeinträchtigen können, wäre sie nicht von einer rehabilitierenden Sequenz gefolgt gewesen.

\section{Fazit}

Trotz der geringen Anzahl der instabilen Einheiten im Talkradiokorpus konnten ihre Auffälligkeit und ihre Resonanz in der Interaktion am diskursiven Umfeld gemessen werden.

Die Instabilität der Idiome erweist sich meistens als eine extrinsische, kontextbedingte Eigenschaft, die eher auf die Instabilität des umgebenden Diskurses zurückzuführen ist. Die untersuchten instabilen Idiome zeigen einerseits, dass meistens zwei Strukturen syntaktisch oder semantisch konkurrieren und/oder dass ein erstes kommunikatives, z.B. rein informationelles, Ziel durch ein anderes, z.B. sprachspielerisches, ersetzt wird.

Allem Anschein nach stören sie die Interaktion nicht wirklich, da ihr Verstehen fast nie thematisiert wird. Nur die Akzeptanz der Formulierung wird ausgehandelt. Dieselben Eigenschaften gelten auch für stabile Idiome. Selbst wenn das lexikalische Material nicht prototypisch verwendet wird und Abweichungen aufweist, sind die instabilen Idiome noch lange nicht absurd. Ihr Zustandekommen kann meistens logisch nachvollzogen werden. Ob in einer stabilen oder instabilen Form scheint also die idiomatische Natur der Einheiten im Fokus der Aufmerksamkeit zu stehen. 


\section{Literatur}

Brown, Penelope/Levinson, Stephen C. (1987): Politeness. Some universals in language usage. (= Studies in interactional sociolinguistics). Cambridge, UK: Cambridge University Press.

Burger, Harald (2015): Phraseologie. Eine Einführung am Beispiel des Deutschen. 5., neu bearb. Aufl. (= Grundlagen der Germanistik 36). Berlin: Schmidt.

Dobrovol'skij, Dmitrij/Piirainen, Elisabeth (2005): Figurative language. Cross-cultural and cross-linguistic perspectives. (= Current research in the semantics/pragmatics interface 13). Amsterdam: Elsevier.

Dobrovol'skij, Dmitrij/Piirainen, Elisabeth (2009): Zur Theorie der Phraseologie. Kognitive und kulturelle Aspekte. (= Linguistik 49). Tübingen: Stauffenburg.

Duden (2013): Duden 11: Redewendungen. Wörterbuch der deutschen Idiomatik. 4., neubearb. u. aktual. Aufl. Berlin: Dudenverlag.

Espinat, Marine (2015): L'emploi d'unités polylexématiques idiomatiques à l'oral - Aspects linguistiques et psycho-cognitifs (corpus allemand et français). Paris: Université ParisSorbonne. [Dissertation].

Espinat, Marine (i.Dr.): Des idiomes sur les ondes: La stabilité dans le discours mouvementé?. In: Gautier, Laurent (Hg.): Perspectives franco-allemandes sur le figement. (= Studien zum deutsch-französischen Diskursvergleich 3). Frankfurt a.M.: Lang.

Goffman, Erving (1967): Interaction ritual. Essays on face-to-face behavior. New York: Doubleday.

Grice, Paul (1975): Logic and conversation. In: Cole, Peter/Morgan, Jerry L. (Hgg.): Speech acts. (= Syntax and semantics 3). New York: Academic Press. 41-58.

Techtmeier, Bärbel (2001): Form und Funktion von Metakommunikation im Gespräch. In: Brinker, Klaus et al. (Hgg.): Text- und Gesprächslinguistik. Bd. 2. (= Handbücher zur Sprach- und Kommunikationswissenschaft 16). Berlin /New York: De Gruyter. 1449-1463. 
